\title{
The Education of Human Capital and the Development of Urban Economic Resilience
}

\author{
Chen Yiwei ${ }^{1, *}$ Wang $\mathrm{He}^{2}$ \\ ${ }^{1}$ School of economics and management, Southeast University, Jiangsu Province, Nanjing 211189 \\ ${ }^{2}$ College of civil engineering, Southeast University, Jiangsu Province, Nanjing 211189 \\ ${ }^{*}$ Corresponding author. Email:1179317399@qq.com
}

\begin{abstract}
As an important source of urban innovation, human capital is the key to enhance the resilience of urban economy. Therefore, the education of human capital plays a very important role in the development of cities. Based on the patent data of 264 prefecture level cities in China and the urban population scale of prefecture level cities in China, this paper studies the impact and mechanism of human capital education on urban economic resilience. The results show that the higher the level of human capital, the higher the level of urban economic resilience.
\end{abstract}

Keywords: human capital education; urban economic resilience; industrial agglomeration; population scale; industrial innovation

\section{INTRODUCTION}

In the process of improving urban economic resilience, in order to encourage industrial innovation and promote the invention of new technology in the industry, the cultivation and education of human capital is very important. But throughout the existing literature, the existing literature respectively from the financial, employment, legal and other aspects of human capital research, but the research on the relationship between human capital education and urban economic resilience is relatively small. Audia and Goncalo (2007) found that creative thinking is the key to continuous innovation in the computer software development industry. The more creative the industry, the more competitive it is in the market. Kapoor and lim (2007) found that the change of individual emotions will affect the innovation efficiency of enterprises through business merger and acquisition events. [1] Liu et al. (2017) through the research on the patent data of the city, found that the level of human capital plays a very important role in the innovation of the industry. [2] It can be seen that the existing literature mainly studies the impact on industry innovation from the perspective of human capital, while the research on urban economic resilience from the perspective of human capital is still relatively lacking, and less literature studies the relationship between industrial agglomeration, human capital and urban economic resilience. Therefore, the research on urban economic resilience from the perspective of human capital is helpful to supplement and improve the city Research on the resilience of city economy.

Regional factors are the main factors that affect the distribution of human capital and the resilience of urban economy. From a regional perspective, China's rapid economic growth and the improvement of urban economic resilience are not balanced, and some regions also pay a high environmental cost in the process of economic development. In this paper, from the perspective of invention patents, we verify the impact of human capital education on urban economic resilience, and take the number of top scholars in Ming and Qing Dynasties of each prefecture level city in China and the number of school relocation in each prefecture level city in 1950 as the tool variable of human capital item, and the wall area of each prefecture level city in Ming and Qing Dynasty as the tool variable index of urban agglomeration degree as the tool variable To eliminate the impact of the environment on industrial agglomeration, in-depth study of the impact of human capital education on urban economic resilience and its specific mechanism of action, to provide a large sample of empirical evidence for the promotion of urban economic resilience and human capital related research.

\section{LITERATURE REVIEW AND RESEARCH HYPOTHESIS}

\subsection{Literature review}

Human capital is the key factor of industry innovation. The existing literature mainly studies the characteristics of human capital from enterprises and individuals. Wall (2013) believes that the education level of labor force is the key factor to determine a company's acceptance of new technology. [4] Hill (2012) thinks that the reason why many enterprises can't recover to the original level of development after experiencing a big external shock is the massive loss of labor force. [6] Drobniak (2012) believes that the faster an enterprise accepts new things, the less affected it will be by external shocks. [6] According to 
Zhang Wenqing (2019) and tkinson (1969), enterprises can absorb new technologies through independent innovation, so as to improve their production efficiency. [7] Xuanchangyong, Tang Chengwei and Yan Weilong (2012) believe that regions with low human capital level can achieve regional industrial upgrading through OEM and technology spillover effect. [8] Yang Jun, LV Linguang and Luo Ying (2007) believed that a city with a high level of human capital is the source of a city's industrial structure innovation and stable development of economic growth, and the level of human capital in different regions is different. [9]

The existing literature also gradually finds out the impact of external environment on urban human capital. A good external environment is conducive to the individual's health, thus contributing to scientific research innovation and individual behavior decision-making. On the other hand, the pollution of the external environment may lead to the loss of high-quality talents, and they will develop to cities with better environment, which is not conducive to the occurrence of industrial innovation. Higdon et al (2015) investigated a large refinery in Mexico and found that every $1 \%$ increase in sulfur dioxide in the air will lead to a $0.61 \%$ decrease in working hours of employees. When a large refinery is shut down, for every $20 \%$ decrease in the concentration of sulfur dioxide in the air, the working hours of employees will increase by $4 \%$. At the same time, air pollution will also affect students' attendance. [10] The working hours of employees and the attendance of college students will have a positive and significant impact on the number of scientific research results, so the external environment will have an impact on the change of urban scale and urban economic resilience, which is also the reason why this paper chooses air PM2.5 index as the tool variable of industrial agglomeration.

At the same time, through the past development experience, we can also draw the conclusion that even the two cities with similar human capital level will have different economic development levels due to different urban population size and geographical location levels. Temple (1999) compares Brazil, China and India. Its population scale and human resource level are close, but the gap between the economic development level and technical level of the three major developing countries is quite obvious, which is worth exploring. And the South Korean government has invested a lot of fiscal revenue in education, but the economy has not improved significantly as expected, which may be related to the location distribution of the country.

\subsection{Research hypothesis}

To sum up, this paper proposes: Hypothesis 1: under the same other conditions, the distribution of human capital is related to the population size and location of the city, which will affect the level of economic resilience of the city.

The level of human capital in big cities is higher than that in small and medium-sized cities, and their economic resilience is also better than that in small and medium-sized cities. The level of human capital in eastern cities is higher than that in central and western cities, so the level of economic resilience in eastern cities is also higher than that in central and western cities. Ye Chen and Chen Yong'an (2018) believe that most high productivity enterprises are capital intensive and technology intensive, so they have greater demand for high-quality and high skilled talents. [11] The human capital effect brought by the city scale mainly refers to the large and medium-sized cities gathering a large number of human capital, while the middle and low-level talents are squeezed out by the high cost of living. Large scale cities gather a large number of high-quality human capital, which promotes the probability of enterprises to transform scientific research results into actual production. At the same time, the gathering of highly skilled talents also increases the information spillover and knowledge spillover effects within the industry, between industries or between enterprises, which will be conducive to the improvement of urban production efficiency, and thus can effectively improve the level of urban economic resilience. At present, cross industry innovation does exist in the eastern and central cities, especially in the eastern cities. Generally speaking, the higher product location entropy in the adjacent areas of the industry will increase the probability of the innovation level of the industry, thus improving the economic resilience level of the city. Based on the existing technology and industry, the eastern region needs to strengthen the relevance of industry in order to improve the probability of industrial innovation. Due to the superior geographical location of eastern cities, relatively convenient traffic conditions, the division of labor between industries is relatively clear. However, the market conditions of western cities are backward, the industrial development is relatively single, and the degree of regional integration is not as good as the development of the East and the west, so the ability of urban economic resilience is not as good as that of the East and the central regions.

Hypothesis 2: under the same conditions, the higher the level of human capital, the stronger the resilience of urban economy.

This paper studies the relationship among industrial agglomeration, human capital and urban economic resilience. Drawing lessons from MRW model (1992), this paper constructs the following econometric model: resilience $_{z, t}=$ aresilience $_{z, t-1}+\beta$ nonoagr $_{z, t}+$ cihcl $_{z, t}+r X_{z, t}+u_{z}+\xi_{z, t} \quad(6)$ The resilience of urban economy in this paper is expressed by resilience, T-1 represents the lagged one-stage variable of urban economic resilience, and the lagged one-stage variable is selected to illustrate the sustainability of the variable; nonagr represents the degree of industrial agglomeration, where we use urban population to replace the industrial agglomeration; $\mathrm{HCl}$ represents the level of human capital, which is expressed by the total number of urban patent authorizations; X represents other control variables, which is selected in this paper The ratio of the first industry to GDP, the ratio of the third industry to GDP, the 
ratio of FDI to GDP and the ratio of fixed asset investment to GDP are four indicators. U represents the fixed effect of the city and represents the error term. The subscripts $Z$ and $t$ of each variable in the model (1) represent a city $\mathrm{Z}$ and time $\mathrm{t}$ respectively. [17]

\section{EMPIRICAL CALCULATION}

\subsection{Descriptive statistics}

In descriptive statistics, the mean value of urban economic resilience is -1.36 , and the standard deviation is 5.13 , This shows that after the financial crisis in 2008 , each city has been impacted in different degrees in the development process. Compared with other variables, the distribution of urban economic resilience in the whole country is relatively flat; the average value of industrial agglomeration is 4.531 million, the standard deviation is 309 , and the population distribution of each city is also quite different. The average value of human capital is 63274, and the standard deviation is 121984 , which shows that the industrial innovation ability of each city is also different. The characteristics of other control variables are basically consistent with Wang Shiping and Qian Xuefeng (2018). [18]

\subsection{Regression analysis}

The correlation among industrial agglomeration, human capital and urban economic resilience is regressed. Table 2 reports the OLS benchmark regression results of this paper. In column (1) of Table 2, only lag phase variables, industrial agglomeration degree, human capital level, year and urban fixed effect are added. The regression results show that the regression coefficient of industrial agglomeration is significantly positive, and the urban economic resilience will increase by 0.0006 percentage points for each percentage point increase in the degree of agglomeration. The item of human capital is significantly positive. For each percentage point increase in the item of human capital, the urban economic resilience will increase by 3.04 percentage points. In column (2), the combination of all the control variables is added, and the coefficient symbol is not changed, which shows that besides controlling other factors, the impact of industrial agglomeration on urban economic resilience is still significantly positive, and the impact of human capital on urban economic resilience is still positive. After adding the second term of urban scale and human capital in the third column, it is found that the second term of industrial agglomeration degree is not significant, while the relationship between human capital and urban economic resilience is not linear. However, there may be a positive U-shaped relationship between human capital and urban economic resilience, and the inflection point is $(0.53,1.11)$. This shows that human capital has a negative effect on urban economic resilience in a certain range. When it exceeds the inflection point $(0.53,1.11)$, human capital has a positive effect on urban economic resilience. At the same time, the sign of the regression coefficient of the control variable is basically the same as the expected sign.

\section{SUMMARY}

Based on the large sample data of 264 prefecture level cities in China from 2004 to 2018, this paper uses the difference between the real GDP growth index of each prefecture level city and the real GDP growth index of the city in 2008 to express the urban economic resilience. The population of each city in each year is used to represent the industrial agglomeration item, and the total number of patent authorizations in each city in each year is used to represent the human capital item. The research shows that the industrial agglomeration will promote the improvement of urban economic resilience. The relationship between human capital and urban economic resilience is not a simple positive term, but a positive U-shaped relationship. The turning point is $(0.53$, 1.11).

There are many shortcomings in this paper. In the future, we can carry out in-depth research in the following aspects: first of all, we can expand the source of data, on the basis of the city statistical yearbook, we can pay more attention to the job site to obtain talent information, and further extend other characteristic variables. On the other hand, human capital can be divided into different levels and occupations to judge the impact of different levels and occupations on urban economic resilience.

\section{REFERENCES}

[1] kapoor, R., and K. Lim. The impact of Acquisitions on the Productivity of Inventors at Semiconductor Firms: A Synthesis of Knowledge-Based and Incentive-Based Perspectives[J]. Academy of Management Journal ,2007, 50(5):1133-1155

[2] Liu,H., and A. Salvo.Severe Air Pollution and Child Absences When Schools Can Respond [R].SSRN Working Paper,2018.

[3] Liu,H., and A. Salvo.Severe Air Pollution and Child Absences When Schools Can Respond [R].SSRN Working Paper,2018.

[4] Wall H J., 2013, "The Employment Cycles of Neighboring Cities",Regional Science and Urban Economics,43(1),PP.177-185.

[5] Hill E,St Clair T, Wial H,et al.,2012,"Economic Shocks and Regional Economic Resilience",Building Resilient Regions:Urban and Regional Policy and Its Effects,4,pp.193-274.

[6] Drobniak A.,2012,“The Urban Resilience-Economic Perspecive",Journal of Economic\&Management ,University of Economics in Katowice,10,PP.5-20. 
[7] Zhao Jing Ping Ping. A study on the mental health level and influencing factors of junior students [J]. Chinese Journal of mental health, 1987, 4

[8] Wang Jianzhong. A comparative study of UPI and SCL-90. Chinese Journal of mental health, 1995, 3

[9] Zhang Xiaozhou et al. Investigation report on the psychological quality of college students in Sichuan Province (1) [J]. Sichuan psychological science, 1997, 2

[10] Liu Ying. A study on the mental health of students in Chengdu Institute of physical education. Sichuan psychological science, 1997, 2

[11] Zhang Zhiyong, Luo Shanhong. A comparative study on the results of College Students' SCL-90 scale [J]. Chinese Journal of mental health, 1998, 2

[12] Zhai Hong, et al. A study on the multidimensional structure of College Students' mental health [J]. Chinese Journal of clinical psychology, 2002, 3

[13] Ryff , C.D (1989a) .beyond Ponce de Leon and life satisfaction : New directions i n Cuest of successf ul agi ng $[\mathrm{J}]$. International Journal of behavioral

Development , $12,35-5$ 